\title{
Phthalocyanine Polymers II. Synthesis and Characterization of Fused Metal Phthalocyanine Polymers
}

\author{
Ming Sy $\mathrm{LIAO}^{\dagger}$ and Kung Tu KuO* \\ Department of Chemical Engineering, Ta Hwa College of Technology, \\ Hsin-Chu, Taiwan 307, Republic of China \\ * Institute of Chemical Engineering, National Central University, \\ Chung-Li, Taiwan 32054, Republic of China
}

(Received February 1, 1993)

\begin{abstract}
Fused metal phthalocyanine polymers with higher molecular weight were prepared by baking equal molar quantities of pyromellitic dianhydride and phthalic anhydride with urea, ammonium chloride, ammonium molybdate and various metal sulfates. The structure of the polymers were investigated by comparison with their corresponding metal phthalocyanine derivatives. The elemental, I.C.P., spectra, electrical measurements and X-ray diffraction pattern were made to characterize the polymers. The conductivities of the polymers are in the range of $10^{-4}-10^{-6} \mathrm{Scm}^{-1}$.

KEY WORDS Fused Metal Phthalocyanine / Synthesis / Metal Phthalocyanine Derivatives / Spectroscopic Study / Conductivity / X-Ray Powder Diffraction Pattern /
\end{abstract}

Polymeric phthalocyanines have attracted attention recently for their unusual properties, such as semi-conductivity, photoconductivity, catalytic properties and thermal stability. Several types of phthalocyanine polymers have been prepared, which include (1) those having the phenylene rings of adjacent phthalocyanine structures connected by single bonds in the manner of biphenyl ${ }^{1,2}$; (2) those in which phthalocyanines are joined together by substituents attached to the phenylene ring ${ }^{3,4} ;(3)$ cofacial polymers in which the phthalocyanine units are connected (stacked) together through the central metallic atom ${ }^{5-9}$; (4) polymers that share phenylene rings of the phthalocyanines in fused type $\mathrm{e}^{10-16} ;$ (5) those which phthalocyanines is a pendant group on other polymers (such as polystyrene, Polyacrylamide, etc. $)^{17-20}$; and (6) polymers that contain a phthalocyanine skeleton in their structure ${ }^{21,22}$ to form a long conjugate system all through the main polymer chain.

Marvel $^{23,24}$ and Berezin ${ }^{25}$ prepared type (4) fused copper phthalocyanine polymers by reaction of pyromellitic dianhydride (PMDA), phthalic anhydride (PA), metal salt, ammonium chloride, urea and a molybdate catalyst. The products were chainlike copper containing polymers (repeating unit $n<6$ ). Although individual units in the phthalocyanine structure may not be so symmetrically arranged, it was

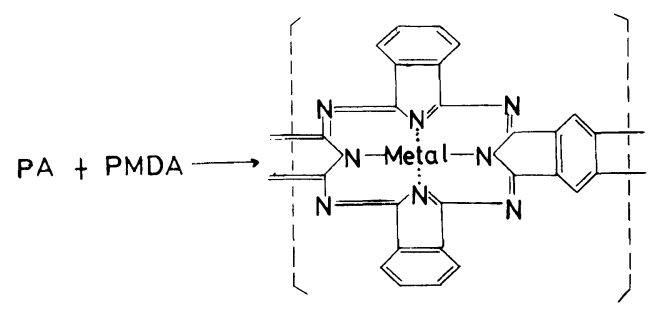

Scheme 1.

† To whom correspondence should be addressed. 
hoped to obtain a polymer with the recurring unit shown in scheme 1 by using equal molar quantities of PMDA and PA. In our previous work, ${ }^{26}$ conditions for preparing fused copper phthalocyanines polymers with higher molecular weight were discussed. In the present study, we extended the reaction to synthesize various fused metal phthalocyanine polymers, and characterized the polymers by UV/Vis, Infrared, elemental analysis, Inductively-Coupled Plasma Atomic Emission Spectrometer (I.C.P.-A.E.S.), X-ray powder diffration spectra and conductivity measurement.

\section{EXPERIMENTAL}

\section{Synthesis}

The method for fused metal phthalocyanine polymers has been reported elsewhere. ${ }^{26}$ The general procedures are as follows:

Pyromellitic dianhydride $(0.02 \mathrm{~mol})$, phthalic anhydride $(0.02 \mathrm{~mol})$, nickel sulfate $(0.0105$ mol), ammonium chloride $(0.041 \mathrm{~mol})$, and on excess of urea are finely ground with a catalytic quantity of ammonium molybdate. The solid mixture is sealed in a glass packed stainless autoclave, and backed at $180^{\circ} \mathrm{C}$ for 4 hours, then $350^{\circ} \mathrm{C}$ for 12 hours. The resulting solid is triturated and washed with water, then methanol. The residue is consecutively extracted with DMF and then acetone in a Soxhlet extraction apparatus for 12 hours, and extracted by $100 \mathrm{ml}$ of 1-chloronaphthalene at $50^{\circ} \mathrm{C}$ for 12 hours. The product is then heated at $c a .300^{\circ} \mathrm{C} / 10^{-3}$ Torr for 12 hours.

\section{Analysis}

Analysis of metal content in the polymers was made by treating them with $1 \mathrm{ml}$ of concentration nitric acid and $0.5 \mathrm{ml}$ of concentrated sulfuric acid in a crucible at high temperature, then diluting with water, and measured by using a Simultaneous I.C.P.A.E.S. (Allied Analytical Systems, Model ICAP 9000). Infrared spectra are recorded in $\mathrm{KBr}$ pellets on a JASCO 810 spectrophotom- eter. UV/Vis spectra is measured using a GuideWave spectrophtometer Model 200. Elemental analysis ( $\mathrm{C}, \mathrm{H}$, and $\mathrm{N}$ ) was made by using a PERKIN-ELMER Elemental Analyzer. A Seimens D-500 X-ray diffractometer is used to study the X-ray pattern of the polymeric samples using the following conditions: target $\mathrm{Cu}$ (Ni-Filter); voltage $40 \mathrm{kV}$; current $30 \mathrm{~mA}$; time constant 4; channel width 0.8 , channel center 1.0. A Two-electrode technique of conductivity measurement is employed using a Keithley 617 electrometer, on samples compressed into discs of $1.3 \mathrm{~cm}$ dia. and $0.1-$ $0.15 \mathrm{~cm}$ in thickness under 9 tons force. A thin conducting silver paint is coated on both sides of the disc and electrical contacts to the sample disc are made by using the same silver paint on the electrodes. The resistance of the samples is measured in a well-shielded oven cycled between 25 and $210^{\circ} \mathrm{C}$ under ambient atmosphere.

\section{RESULTS AND DISCUSSION}

\section{Preparation and Purification}

The polymeric products of PMDA/PA after reacting with various metal sulfates were investigated in detail. The optimum conditions obtained for the synthesis of the fused copper phthalocyanine polymer ${ }^{26}$ are used. The reaction conditions of the present studies are equimolar quantities of pyromellitic dianhydride and phthalic anhydride at $180^{\circ} \mathrm{C}$ for 4 hours and then at $350^{\circ} \mathrm{C}$ for 12 hours.

All the polymers are powders and vary in color from dark blue to black with a metallic luster depending on the reaction condition and metal salt used. The procedures of purification are as follows:

1. Removal of starting material - the crude product is ground and washed by $\mathrm{H}_{2} \mathrm{O}$ and $\mathrm{MeOH}$.

2. Removal of monomers and oligomersthe product is extracted with DMF, acetone and 1-chloronaphthalene.

3. Elimination of end group - the product 
is treated with heating under vacuum.

IR analysis is made to characterize the structure of the product in each step of purification. The first step of purification is to separate the starting materials from the product and the second step removes metal phthalocyanines and metal phthalocyanine oligomers $(n<6)$. The higher molecular weight fused metal phthalocyanine polymers $(n>6)$ are obtained after purification. Additional heating under vacuum treatment eliminates the end groups of the polymers. The yields of polymers are $75.54-87.78 \%$ (Table I). The quantities of extracted metal phthalocyanines and metal phthalocyanine oligomers are also listed in Table I, and the data of elemental analysis are shown in Table II.

\section{Spectroscopic Data}

Infrared Spectra. The polymers, oligomers and phthalocyanine monomers were identified

Table I. Yields of fused polymers

\begin{tabular}{lcccc}
\hline & \multicolumn{2}{c}{ Yield } & $\begin{array}{c}\text { 1-Chloronaphthlene } \\
\text { soluble } \\
\text { fraction }\end{array}$ & $\begin{array}{c}\text { DMF } \\
\text { soluble } \\
\text { fraction }\end{array}$ \\
\cline { 2 - 3 } & & $\%$ & $\%$ \\
\hline PolyMnPc & 87.27 & 4.71 & 4.36 \\
PolyFePc & 87.78 & 6.96 & 5.69 \\
PolyCoPc & 87.57 & 4.29 & 4.32 \\
PolyNiPc & 75.54 & 7.48 & 7.42 \\
PolyCuPc & 86.23 & 6.81 & 6.02 \\
PolyZnPc & 86.21 & 3.32 & 3.75 \\
\hline
\end{tabular}

as described in the previous report ${ }^{26}$ by IR spectra. The oligomers $(n<6)$ of the DMF soluble fraction are similar to the corresponding lower molecular weight metal phthalocyanine derivatives, and show higher intensity than the polymers on IR spectra. Comparing to the IR and UV/Vis spectra, the 1chloronaphthalene soluble fractions of products were identified as metal phthalocyanines.

There is different characteristic pattern on IR spectra for each type of functional group (such as acid, anhydride, imide and amide) in the region of $1600-1800 \mathrm{~cm}^{-1}$. The polymers, followed the purification step 2, show the absorption bands of the - CONHCO- group at 1775 and $1720 \mathrm{~cm}^{-1}$ and no absorptions of other end groups are present in the IR spectra. Thus all polymeric phthalocyanines, obtained

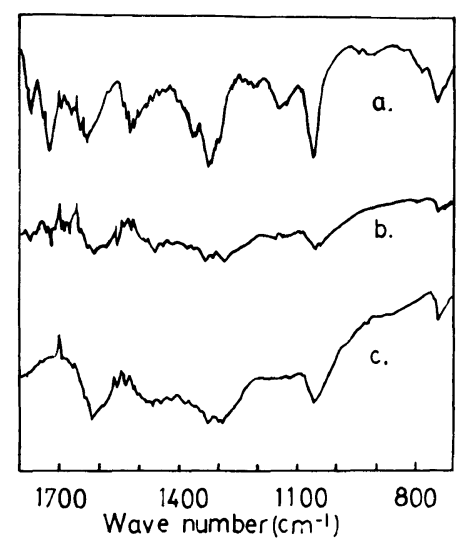

Figure 1. IR-spectra of fused manganeses phthalocyanine polymer (a) DMF soluble fraction; (b) polymer, before vacuum treatment; (c) polymer, after vacuum treatment.

Table II. Elemental analysis of polymers

\begin{tabular}{|c|c|c|c|c|c|c|c|c|}
\hline \multirow{2}{*}{ PolyM-Pc } & \multicolumn{4}{|c|}{ Found } & \multicolumn{4}{|c|}{ Calculated $\left(\mathrm{C}_{26} \mathrm{H}_{10} \mathrm{~N}_{6} \mathrm{M}\right)$} \\
\hline & $\mathrm{C} \%$ & $\mathrm{~N} \%$ & $\mathrm{H} \%$ & $\mathrm{M} \%$ & $\mathrm{C} \%$ & N\% & $\mathrm{H} \%$ & $\mathrm{M} \%$ \\
\hline PolyMnPc & 62.71 & 22.38 & 2.09 & 11.15 & 63.81 & 22.91 & 2.05 & 11.24 \\
\hline PolyFePc & 61.13 & 22.23 & 2.02 & 10.95 & 63.69 & 22.87 & 2.04 & 11.40 \\
\hline PolyCoPc & 62.84 & 21.13 & 1.93 & 11.23 & 63.30 & 22.72 & 2.03 & 11.96 \\
\hline PolyNiPc & 60.65 & 21.54 & 1.87 & 11.18 & 63.33 & 22.73 & 2.03 & 11.91 \\
\hline PolyCuPc & 60.76 & 21.78 & 1.98 & 11.82 & 62.71 & 22.51 & 2.01 & 12.77 \\
\hline PolyZnPc & 61.35 & 22.05 & 1.89 & 12.65 & 62.48 & 22.43 & 2.00 & 13.09 \\
\hline
\end{tabular}


Table III. Infrared absorption data of poly fused metal phthalocyanine polymers

\begin{tabular}{|c|c|c|}
\hline Polymers & IR absorption $\left(\mathrm{cm}^{-1}\right)$ & \\
\hline PolyMnPc & $3545,1610,1545,1320,1290$ & 1048 \\
\hline PolyFePc & $3430,1610,1535,1334,1292,1123$, & $1090,918,740$ \\
\hline PolyCoPc & $3470,6120,1530,1322,1295,1130$, & $1095, \quad 750$ \\
\hline PolyNiPc & $3450,1610,1526,1330,1290,1120$, & $1083,915,732$ \\
\hline PolyCuPc & $3440,1615,1538,1310,1293$ & $1075,910,745$ \\
\hline PolyZnPc & $3450,1620,1532,1305$ & $1070, \quad 750$ \\
\hline
\end{tabular}

Table IV. UN/Vis absorption data of polymers, oligomers and phthalocyanines

\begin{tabular}{|c|c|c|c|}
\hline \multirow{2}{*}{ Polymers } & \multicolumn{3}{|c|}{$\lambda_{\max }(\varepsilon)$} \\
\hline & $\begin{array}{l}\text { 1-Chloronaphthalene } \\
\text { soluble fraction }\end{array}$ & $\begin{array}{l}\text { DMF soluble } \\
\text { fraction }\end{array}$ & Polymer $^{\mathrm{a}}$ \\
\hline PolyMnPc & $724,698,666,642$ (sh), 602,521 & $882,662,358$ & $791(4128)$ \\
\hline PolyFePc & $692,595(\mathrm{sh})$ & $886,678,390$ & $771(5138)$ \\
\hline PolyCoPc & $667,641(\mathrm{sh}), 604$ & $663,601,366$ & $782(6445)$ \\
\hline PolyNiPc & $696(\mathrm{sh}), 668,642(\mathrm{sh}), 603$ & 674,382 & $776(5104)$ \\
\hline PolyCuPc & $609,650(\mathrm{sh}), 676$ & $885,666,430$ & $771(5897)$ \\
\hline PolyZnPc & $695(\mathrm{sh}), 668,639(\mathrm{sh}), 603$ & 680,386 & $775(7354)$ \\
\hline
\end{tabular}

a Solvent used - concentrated sulfuric acid.

Molar absorptivity of polymers were calculated from the phthalocyanine skeleton repeating unit.

according to the synthetic procedures in this paper, have imide end groups. The peripheral anhydride groups of the polymers seem to have been converted to the imide type structure. (Figure 1).

The end groups of the product through the last purification, additional thermal and vacuum treatment, are eliminated.

Polymers are characterized as similar shape on IR spectra and contain absorption bands assignable to the phthalocyanine skeleton in the range of $732-750,1048-1095,1290$ $1330,1526-1545,1610-1625$, and 3470 $3545 \mathrm{~cm}^{-1}$ (Table III). The spectra of the polymers show less fine structure and lower intensity of the bands compared with the spectra of their corresponding low molecular weight analogues.

UV/Vis Spectra. The UV/Vis absorption bands for the DMF and 1-chloronaphthalene soluble fractions of product mixture are listed

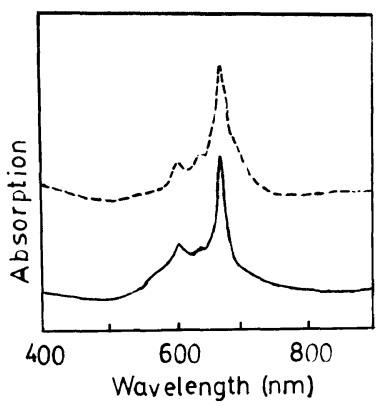

Figure 2. Electronic absorption spectra (in 1-chloronaphthalene) (a) nickel phthalocyanine (----); (b) 1chloronaphthalene soluble fraction of fused nickel phthalocyanine polymer (-).

in Table IV. The DMF soluble fractions of the crude product are absorbed in the region of $663-703 \mathrm{~nm}$. The extracted fractions of product in 1-chloronaphthalene have the same absorption bands as the corresponding monomer metal phthalocyanines. (Figure 2). The 1chloronaphthalene soluble fractions of product mixture, by comparing IR, ${ }^{28} \mathrm{UV} / \mathrm{Vis}$ 
spectra and X-ray powder diffraction pattern ${ }^{29}$ with previous report, were identified as alpha type metal phthalocyanines.

The UV/Vis absorption spectra of fused metal phthalocyanine polymers are recorded by using concentrated sulfuric acid as the solvent (Table IV). All the polymers show maximum absorption wavelengths at 206 208, 294-304, and 771-791 nm similar to the analogous monomer metal phthalocyanines. The absorptions of the polymers are broader and less intense than the absorption of the corresponding monomers. The molar absorptivity $(\varepsilon)$ of the polymers, as determined from the repeating phthalocyanine skeleton, are also shown in Table IV. The dark blue color of the polymers maybe due to $\mathrm{pi} \rightarrow \mathrm{pi}^{*}$ transitions. Absorption bands due to this transition appeared at longer wavelength than the parent metal phthalocyanines.

\section{$X$-Ray Powder Diffraction}

The X-ray powder diffraction patterns of the polymers show qualitatively similar spectrographs in the range of $0-90$. The X-ray data of poly phthalocyanines, containing metal with $\mathrm{Cu}, \mathrm{Co}$, and $\mathrm{Ni}$, have been made by Achar. ${ }^{27}$ Interplanar spacings $(\AA)$ were in the range 13.88-13.07 and 3.11-3.22. In our work, two

Table V. X-Ray powder diffraction data of polymers ${ }^{\mathrm{a}}$

\begin{tabular}{ccc}
\hline Polymer & $2 \theta$ & $\begin{array}{c}\text { Interplaner spacing } \\
\AA\end{array}$ \\
& & 7.285 \\
PolyMnPc & 12.143 & 3.317 \\
& 26.868 & 7.227 \\
PolyFePc & 12.241 & 3.420 \\
& 26.044 & 7.419 \\
PolyCoPc & 11.923 & 3.317 \\
PolyNiPc & 26.868 & 7.180 \\
& 12.321 & 3.374 \\
PolyCuPc & 26.400 & 7.814 \\
& 11.318 & 3.312 \\
PolyZnPc & 26.903 & 7.890 \\
& 11.209 & 3.378
\end{tabular}

a $\lambda=0.1541 \mathrm{~nm}=2 d \sin (\theta)$. peaks observed for all the polymers, one sharp peak with maximum intensity and the other one not well resolved peak broadened with less intensity. The values of interplanar spacings and diffraction angles are listed in Table $\mathrm{V}$. The shapes of the X-ray diffraction spectrographs indicate that these polymers have different crystallinity from the corresponding metal phthalocyanines.

\section{Conductivity Measurement}

The conductivity of fused metal phthalocyanine polymers with temperature are plotted in $\sigma$ versus $1 / T$ (Figure 3) according to the following expression:

$$
\sigma=\sigma_{\mathrm{o}} \exp \left(-E_{\mathrm{a}} / 2 k T\right)
$$

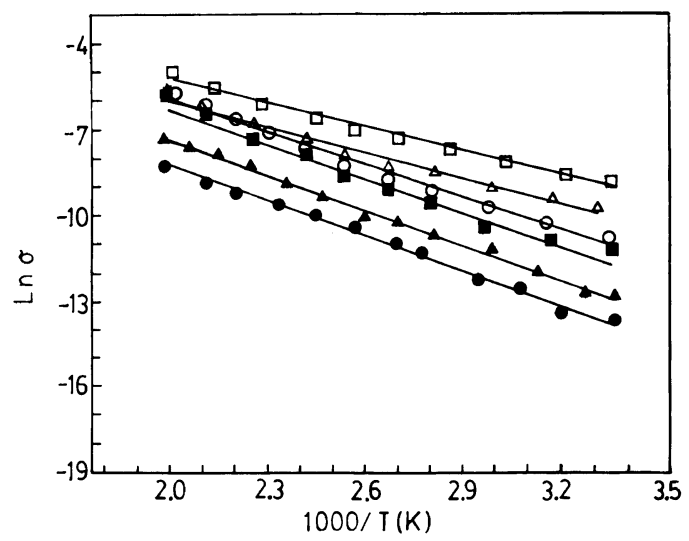

Figure 3. Conductivity versus temperature plot of fused metal phthalocyanine polymers (a) polyCuPc, $\square$; (b) polyMnPc, $\triangle$; (c) polyZnPc, $\bigcirc$; (d) polyCoPc, $\mathbf{\square}$; (e) polyNiPc, $\mathbf{\Delta}$; (f) polyFePc,

Table VI. Electrical properties of polymers

\begin{tabular}{lccc}
\hline Polymer & $\sigma_{\mathrm{rt}} / \mathrm{S} \mathrm{cm}^{-1}$ & $\sigma_{\mathrm{o}} / \mathrm{S} \mathrm{cm}^{-1}$ & $E_{\mathrm{a}}(\mathrm{eV})$ \\
\hline PolyMnPc & $1.46 \times 10^{-4}$ & 1.189 & 0.526 \\
PolyFePc & $5.98 \times 10^{-6}$ & 0.882 & 0.702 \\
PolyCoPc & $3.60 \times 10^{-5}$ & 4.926 & 0.684 \\
PolyNiPc & $2.59 \times 10^{-6}$ & 2.232 & 0.699 \\
PolyCuPc & $1.52 \times 10^{-4}$ & 1.245 & 0.468 \\
PolyZnPc & $3.79 \times 10^{-5}$ & 5.128 & 0.652 \\
CuPc, NiPc, & $10^{-11}-10^{-15}$ & & $1.4-1.9$ \\
FePc & & &
\end{tabular}

a Literature data. ${ }^{1,21}$ 
where $E_{\mathrm{a}}$ is the width of the forbidden energy band or energy gap, $\sigma$ is the specific conductivity at $T / \mathrm{K}, \sigma_{\mathrm{o}}$ is the conductivity as $T$ approaches infinity and $k$ is the Boltzmann's constant. The conductivities and energy gaps, determined by the linear least square fit to the above expression, are presented in Table VI. The conductivities of the polymers $\left(10^{-4}\right.$ $10^{-6}$ ) at room temperature are greater than the corresponding monomer metal phthalocyanines $\left(10^{-13}-10^{-11} \mathrm{~S} \mathrm{~cm}^{-1}\right)^{30,31}$ and oligomers $\left(10^{-9} \mathrm{~S} \mathrm{~cm}^{-1}\right)^{32}$ by several order. Interparticle contact resistance and overall crystallographic orientations in the fused metal phthalocyanines should differ from parent metal phthalocyanines because of the greater planarity due to fused structures. The extension of planarity in the polymeric structures may facilitate greater intermolecular contact and interaction of $3 \mathrm{~d}$ orbitals with pi orbitals of the neighboring phthalocyanine units, thus, providing a path for charge carriers to move. The energy gap of fused polymers are in the range of $0.468-0.730 \mathrm{eV}$.

\section{CONCLUSIONS}

Fused metal phthalocyanine polymers are prepared and yields of the polymers are $75.54-87.78 \%$. The peripheral end group of the polymers before vacuum treatment is an imide group in structure. The X-ray diffraction patterns show that the polymers have different types of crystallinity from the corresponding monomer metal phthalocyanine. The conductivities of the polymers are greater than the corresponding monomer metal phthalocyanines and oligomers for several orders. The energy gap of fused polymers are in the range of $0.468-0.730 \mathrm{eV}$.

Acknowledgments. The work reported here was supported by National Science Council, Republic of China, under the project number: NSC79-0405-E008-05.

\section{REFERENCES}

1. B. N. Achar, G. M. Fohlen, and J. A. Parker, J. Polym. Sci., Polym. Chem. Ed., 25,443 (1987).

2. A. A. Berlin and A. I. Sherle, Inorg. Macromol. Rev., 1, 235 (1971).

3. H. Shirai, K. Kobayashi, Y. Takemae, A. Suzuki, O. Hirabaru, and N. Hojo, Makromol. Chem., 180, 2073 (1979).

4. B. N. Achar, G. M. Fohlen, and J. A. Parker, J. Polym. Sci., Polym. Chem. Ed., 20, 2773 (1982); ibid., 20, 2781 (1982); ibid., 21, 1025 (1983); ibid., 22, 319 (1984).

5. M. Tsutsui, Gov. Rep. Announce Index (U.S.), 81, 3348 (1981); Chem. Abstr., 96, 14553b.

6. R. S. Nohr, P. M. Kuznesof, K. J. Uynne, M. E. Kenny, and P. G. Siebenman, J. Am. Chem. Soc., 103, 4371 (1981).

7. M. Hanack, K. Mitulla, and O. Schneider, Chemica Scripta, 17, 139 (1981).

8. G. Meyer and D. Wohrle, Materials Science, 7, 265 (1981).

9. T. J. Marks, J. Am. Chem. Soc., 101, 7071 (1979).

10. R. Bannehr, G. Meyer, and D. Worhle, Polym. Bull., 2, 841 (1980).

11. H. Inoue, Y. Kida, and E. Imoto, Bull. Chem. Soc. Jpn., 40, 184 (1967).

12. A. I. Sherle, V. V. Promyslova, N. I. Shapiro, V. R. Esphtein, and A. A. Berlin, Vysokomol. Soedin., Ser. $A, 22,1258$ (1980).

13. R. Bannehr, N. Jaeger, G. Meyer, and D. Wohrle, Makromol. Chem., 12, 2633 (1981).

14. D. Wohrle, Adv. Polym. Sci., 50, 45 (1983).

15. D. Worhle, U. Marose, and R. Knoop, Makromol. Chem., 186, 2209 (1985); D. Wohrle and E. PreBner, ibid., 186, 2189 (1985); D. Wohrle and B. Schulte, ibid., 186, 2229 (1985).

16. B. N. Achar, G. M. Fohlen, and J. A. Parker, J. Polym. Sci., Polym. Chem. Ed., 20, 1785 (1982).

17. J. H. Schutten and J. Zwart, J. Mol. Catal., 5, 109 (1979).

18. J. Zwart and J. H. van Wolput, J. Mol. Catalyst, 5, 3235 (1979).

19. H. Shirai, A. Maruyama, K. Kobayashi, and N. Hojo, J. Polym. Sci., Polym. Lett. Ed., 17, 661 (1979); Makromol. Chem., 181, 575 (1980).

20. T. R. Walton, J. R. Griffith, and J. P. Reardon, J. Appl. Polym. Sci., 30, 2921 (1985).

21. D. Wohrle, G. Kossmehl, and G. Manecke, Makromol. Chem., 154, 111 (1972).

22. M. Gebler and J. Inorg, Nucl. Chem., 43, 2759 (1981).

23. C. S. Marvel and J. H. Rassweiler, J. Am. Chem. Soc., 80, 1197 (1958).

24. C. S. Marvel and M. M. Martin, J. Am. Chem. Soc., 80, 6600 (1958).

25. B. D. Berezin and L. P. Shormanova, Vysokomol. Soyedin., Ser. A, 10, 2, 384 (1968). 


\section{Phthalocyanine Polymer}

26. M. S. Liao and K. T. Kuo, J. Polym. Soc., Polym. Chem., Ed., 28, 2349 (1990).

27. B. N. Achar, G. M. Fohlen, J. A. Parker, and J. Keshavayya, J. Polym. Sci., Polym. Chem. Ed., 25, 443 (1987).

28. A. N. Sidorov and L. P. Kotlyar, Optika $i$ Spektroskopiya, Int. Ed. Eng., 11, 92 (1961).

29. (a) I. Kumano, Denshi Shashin Gakkaishi, 22, 111
(1984); (b) Data file of Seimens X-ray diffractometer (D-500), 37-1846 (1989).

30. J. Blomquist, L. C. Moberg, L. T. Johansson, and R. Larsson, Inorg. Chim. Acta, 53, L39 (1981).

31. B. D. Berezin and A. N. Shlyapova, Vyskomol. Soedin. Ser., A, 15, 1671 (1973).

32. M. S. Liao and K. T. Kuo, Chemistry (Chinese Chem. Soc. Taiwan China), 48, 317 (1990). 